\title{
ANALYSIS OF THE INTERACTION BETWEEN SAFETY AND SECURITY DEMONSTRATED ON A MOBILE ROBOT AND A PRODUCTION NETWORK
}

\author{
Isabella Reithner*1, Maximilian Papa ${ }^{1,2}$, Mohamed Aburaia ${ }^{1}$, \\ Wilfried Wöber ${ }^{1,3}$ \& Clemens Ambros ${ }^{1}$
}
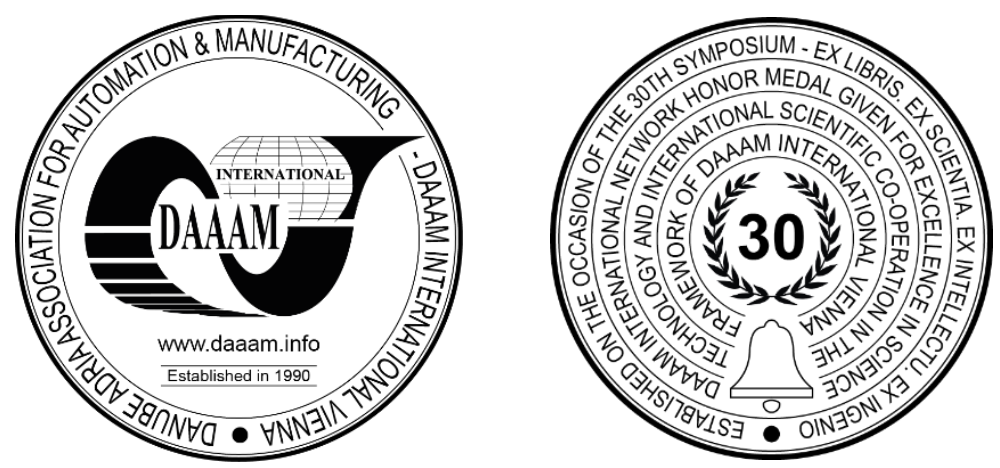

This Publication has to be referred as: Reithner, I[sabella]; Papa, M[aximilian]; Aburaia, M[ohamed]; Woeber, W[ilfried] \& Ambros, C[lemens] (2021). Analysis of the Interaction Between Safety and Security Demonstrated on a Mobile Robot and a Production Network, Proceedings of the 32nd DAAAM International Symposium, pp.0349-0355, B. Katalinic (Ed.), Published by DAAAM International, ISBN 978-3-902734-33-4, ISSN 1726-9679, Vienna, Austria DOI: $10.2507 / 32$ nd.daaam.proceedings.051

\begin{abstract}
The $4^{\text {th }}$ Industrial Revolution increases connectivity within the production environment by transforming traditional automation systems to cyber-physical production systems. This conversion impacts intralogistics of a factory: Intelligent and interconnected transport systems, in the form of autonomous mobile robots, are increasingly integrated into the production process. This enhanced connectivity expands the attack surface of production facilities for cyber-attacks. In addition, the interconnection of safety and security poses a risk for people working on machines or around mobile robots in case of an attack. In order to highlight the interaction between safety and security, this study discusses the requirements of a mobile robot from both perspectives. Verification in practice is achieved by performing a penetration test on a test environment of a production network with an integrated mobile robot. The discussion of the detected vulnerabilities outlines the hazards of operating a mobile robot in an interconnected production network, causing a loss of Confidentiality, Integrity and Availability. Finally, these results are providing a basis for trustworthy mobile robotics implementations in production plants.
\end{abstract}

Keywords: Mobile Robotics; Safety \& Security; Penetration Test; Production Network

\section{Introduction}

The digitalization within production as part of the $4^{\text {th }}$ Industrial Revolution leads to a high degree of connectivity in industry [1]. Existing devices are replaced by Cyber-Physical Production Systems (CPPS) [1] and the material flow within a plant is realized with automated guided vehicles or autonomous mobile robots [2].

\footnotetext{
${ }^{1}$ Department of Industrial Engineering, University of Applied Science Technikum Wien, Vienna, Austria

${ }^{2}$ Institute of Management Science, TU Wien, Vienna, Austria

${ }^{3}$ Institute for Integrative Nature Conservation Research, University of Natural Resources and Life Sciences, Vienna, Austria
} 
This new approach requires a revision of outdated hierarchical communication models such as the Automation Pyramid and the isolation of Information Technology (IT) and Operational Technology (OT), in order to cover all aspects. However, with the increasing connectivity of industrial systems, the attack surface for cyber-attacks affecting CPPS, e.g., mobile robots, is increasing [3]. In 2019, a survey pointed out that 59\% of production companies expected an increase in security incidents [4]. Furthermore, the priorities of IT and OT systems are different, as the protection goals of Confidentiality, Integrity and Availability, also known as the CIA triad, indicate. Confidentiality has the highest priority within an IT system, followed by Integrity of information. Availability is only in third place. However, this ranking is different for an Industrial Automation and Control System (IACS). Here, Availability represents the highest objective, followed by Integrity and Confidentiality [5].

Despite its relevance today, security is not the only issue in robotics. Technical safety measures are intended to reduce threats to functional safety and therefore safety of persons is enhanced. If these technical safety measures are threatened by security vulnerabilities, they can be compromised in case of an attack [5]. Therefore, the two-way interaction between safety and security has recently been recognized as an issue by companies [6][7] and is used as motivation for the following research.

In order to solve the problems mentioned above, the requirements for an application involving mobile robotics in Austria are first analysed followed by a focus on cyber-security. The knowledge acquired is applied to a dedicated demonstrator with a mobile robot by conducting a risk assessment. Subsequently, a vulnerability analysis and selected penetration test procedures are executed on the demonstrator and the mobile robot. In this concrete scenario, the connection between safety and security as well as the impact of security on safety in mobile robotics is to be analysed.

\section{Current Requirements for an Interconnected Production Environment Including Mobile Robotics}

In order to meet the requirements of the $4^{\text {th }}$ Industrial Revolution, it is necessary to shift away from traditional automation systems and focus on a higher degree of flexibility. Custom requirements can be fulfilled using mobile robots, offering a high degree of flexibility in navigation, task performance and organization [8][9]. With this increase in flexibility, a growth phase in the market of mobile robotics is currently evident [10]. However, the implementation of flexible transport systems in interconnected production environments poses a new challenge in the context of security as the focus in robotics has long been on safety, development costs, speed to market and customer characteristics [11]. Requirements are therefore not only placed on the performance but also safety of the implemented vehicles which can be divided into mandatory laws and optional requirements based on the respective application and process.

Figure 1 outlines legal framework conditions with relevance for the development and implementation of mobile robotics in Austria [12]. National legislation defines the basic regulations for this purpose. In Austria, these are the Maschinen-Sicherheitsverordnung 2010 (BGBl. II Nr. 282/2008) ${ }^{4}$, ArbeitnehmerInnenschutzgesetz 1994 (BGBl. Nr. 450/1994) ${ }^{5}$ and Netz- und Informationssystemsicherheitsgesetz 2018 (BGB1. I Nr. 111/2018).

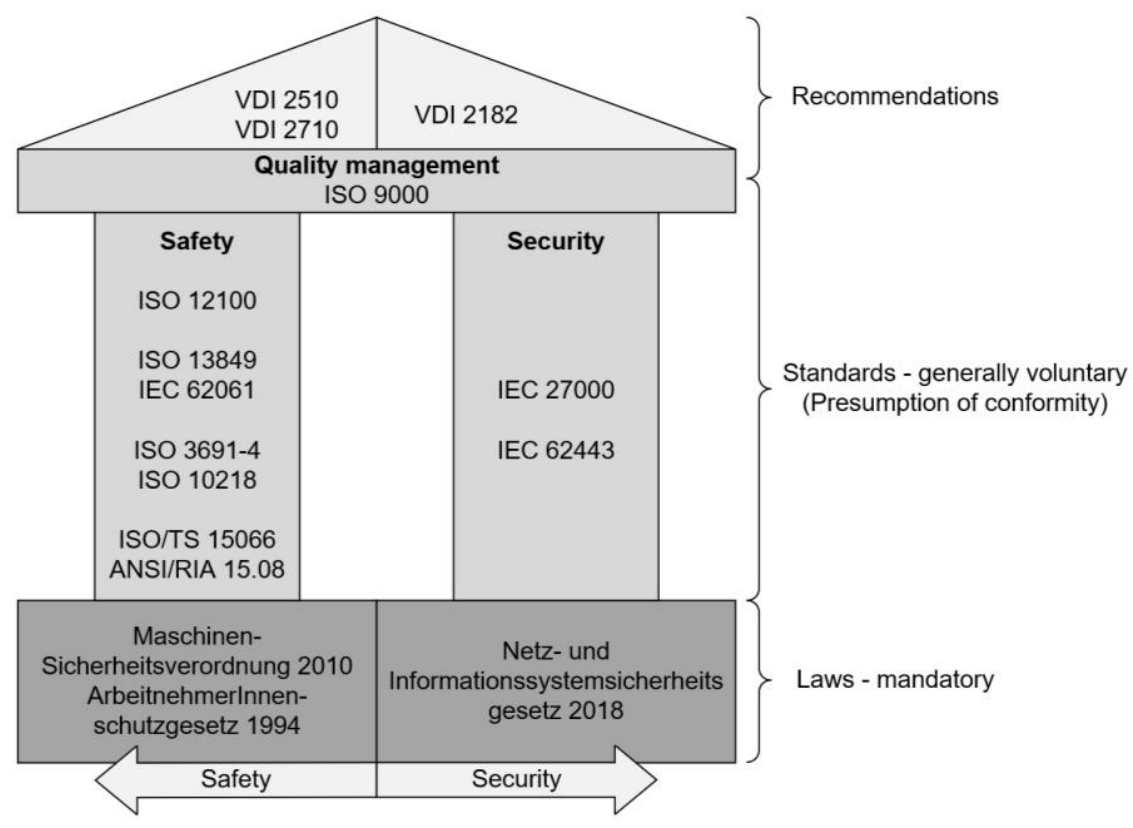

Fig. 1. Selection of relevant laws, standards, and recommendations for mobile robotics in Austria

\footnotetext{
${ }^{4}$ Equivalent EU regulation: Directive 2006/42/EC

${ }^{5}$ Worker Protection Act 1994

${ }^{6}$ Equivalent EU regulation: Directive (EU) 2016/1148
} 
Apart from national laws, international and national standards concerning the current state-of-the-art in safety and security are providing a framework for the operation and marketing of mobile robots. Despite the voluntary nature of standards, compliance may be mandatory by law (e.g., for the presumption of conformity) [13]. The implementation of a quality management mechanism is a recommended way to combine safety and security as the essential aspects for fully automated vehicles are: Quality, safety and security [14]. Furthermore, Figure 1 shows the guidelines of the Association of German Engineers (Verein Deutscher Ingenieure - VDI). These guidelines similarly provide state-of-the-art information elaborated by experts [15].

In addition to legal requirements, key requirements for a mobile robot, identified by a literature study, are Hardware, Navigation, and the Control System. The requirements for the type of the mobile robot [16][17], its geometric dimensions [18], load size [17] as well as the sensor system [19][20] and the power supply [19][18] are grouped in the category Hardware. Navigation includes the use of a guide path [17] and the integrated path planning functionalities [21]. The Control System is closely related to this category. Here, a distinction can be made between a central high-level control system for integrating several mobile robots into a production plant and a low-level control system directly on the vehicle [21]. Within the production process, indicators like idle time [17], average operation time [21], delivery reliability [17] and resource utilization [17][21] are identified as key requirements.

Besides safety, which is covered by laws and standards, security must also be taken into account. Unprotected, highly interconnected CPPS, as mobile robots are, provide increased attack surfaces for cyber-attacks. In addition, a large number of different security threats can amplify vulnerabilities of insufficiently protected CPPS during an attack. Typical security breaches lead to the loss of one of the three security objectives: Confidentiality, Integrity and Availability. But for mobile robotics, there is an additional component: Physical interventions in the environment may result in safety hazards.

The Robot Operating System (ROS) [3], a framework for mobile robots, has not only become established in the research sector, but is starting to develop a stronger position in the industrial sector as well [22]. The advantage of using ROS is the possibility of controlling mobile platforms combined with manipulators (mobile manipulators) in a single software framework [12]. However, there are several vulnerabilities in the implementation ROS such as missing authentication, authorization and encryption procedures [3], making it possible to bypass or deactivate the safety system in the event of an attack [23].

In order to counteract these challenges, a holistic security approach is necessary when designing a system from the ground up (Security by Design). The IEC 62443 series provides seven basic requirements in addition to the security principle Defense in Depth (also called the Zones and Conduits model) which are intended to extend the CIA triad [5]:
1. Access Control:
Passwords and user authentication
2. Use Control:
User roles and authorization enforcement
3. Data Integrity:
4. Data Confidentiality:
Session handling, cryptography
5. Restrict Data Flow:
Encryption
6. Timely Response to Event: Logs
7. Resource Availability: $\quad$ System backup and recovery

\section{Materials and Methods: Analysis of Safety and Security for Mobile Robotics}

In order to practically analyse the cyber-security risks of mobile robotics in terms of safety, a demonstrator is developed. It is a test environment for a production network with an OPC UA server ${ }^{7}$ (Process Control Server), a Human Machine Interface (HMI) and a MiR $100^{8}$ mobile robot. The demonstrator is an enhancement of the system presented in Reithner et al. [24] and the network topology used for the analysis performed is shown in Figure 2.

Compared to the previous version of Reithner et al. [24], the desktop PCs have been replaced by industry-compatible network devices from Siemens AG. These include a Siemens Scalance S615 router and a Siemens Scalance W774-1 access point. The Process Control Server has been implemented with a Revolution Pi Core 3+ 32GB by Kunbus GmbH. Additionally, the OPC UA communication with the robot has been revised. In order to trigger the individual missions already predefined on the MiR100, it is necessary to send a POST request to the REST interface of the MiR100. Since this is not possible directly from the OPC UA server, a Raspberry Pi (MiRWrapper) is used as a gateway.

The demonstrator as described above provides the basis for the following practical analysis of the interaction between safety and security. A risk assessment is performed first, followed by a penetration test and vulnerability analysis.

\footnotetext{
${ }^{7}$ https://github.com/open62541/open62541

${ }^{8}$ https://www.mobile-industrial-robots.com/de/solutions/robots/mir100/
} 


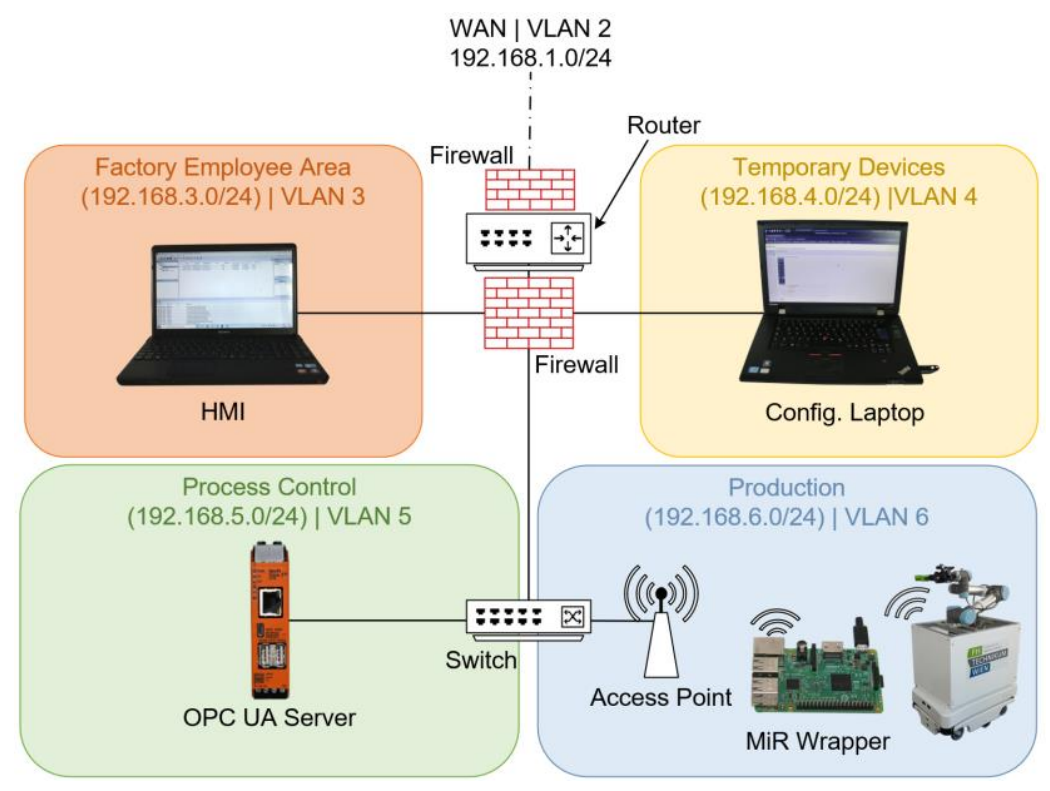

Fig. 2. Zones configured as VLANs at the demonstrator

\subsection{Risk Assessment of the Demonstrator}

The risk assessment follows the process provided by the OVE EN 62443-3-2:2018 [25]. The first step is to identify the assets of the demonstrator, which are listed Table 1. Based on the identified assets and access points, the risks are identified and assessed within a multistage process. The most significant threats to the demonstrator, which may lead to limitations or failures, are identified during a High-Level Risk Assessment. After revising the Zones and Conduits model used for the demonstrator, all threats previously classified as High or Extreme are re-examined in the Detailed CyberSecurity Risk Assessment. In order to assess the security risk of the respective vulnerability, the risk classes identified during the risk assessment are used for the concrete evidence at the demonstrator.

\section{Industrial Automation and Control System (IACS) components:}

- Router [Siemens Scalance S615]

- Switch [CISCO Catalyst 2950]

- Access point [Siemens Scalance W774-1]

- Revolution Pi "Process Control Server" [Revolution Pi Core 3+ 32GB]

- Mobile robot [MiR100]

- Raspberry Pi “MiRWrapper" [Raspberry Pi 3B V1.2]

- Laptop Win10 "HMI" [Sony VAIO PCG-71211M]

- Laptop Win10 “Configuration Laptop” [Lenovo ThinkPad T460p]

\section{Communication infrastructure:}

2.1. Internal dataflow - Protocol:

- OPC UA (between the Process Control Server, HMI and MiRWrapper)

- Hypertext Transfer Protocol (HTTP) (between the MiRWrapper and mobile robot)

2.2. Internal dataflow - Medium:

- LAN (connection of the server, switch, access point, HMI and configuration laptop)

- WLAN (access point, MiRWrapper and MiR100)

2.3. External data flow:

- Access to the internet (only during configuration)

3. Legal position:

- Assurance of functional safety

Table 1. Identified assets concerning the demonstrator 


\subsection{Penetration Test and Vulnerability Analysis executed on the Demonstrator}

The penetration test is executed according to the methodology presented in Shebli \& Beheshti [26] combined with the Open-Source Security Testing Methodology Manual (OSSTMM) [27]. The first step, the preparation, includes the previously mentioned risk assessment, the choice of tools involved and the calculation of the robot's speed. Afterwards, information about the system is gathered and an automatic vulnerability analysis is carried out by OpenVAS ${ }^{9}$. The vulnerabilities found are then exploited to disrupt the operation of the demonstrator or gain access to sensitive information. Tools deployed for this purpose are: hping(3), hydra, kisment(1), netcat(1), nmap(1), tcpdump(8) and Wireshark.

The following limitations became apparent when carrying out the penetration test: The MiR100's safety system was examined during the risk assessment but not considered as a target of the penetration test. The robot poses a potential danger to humans in the case of a successful attack or unsuccessful reversal of the test. Likewise, the MiR100's internal router and computer were not considered a target.

\section{Discussion of the Results}

The results of the risk assessment based on the OVE EN 62443-3-2:2018 [25] indicate a high to extreme cyber-security risk. The classification of $63 \%$ of the risks identified in the Detailed Risk Assessment is High. Another $20 \%$ are considered extreme, as human injury may result. At the same time, none of the risks are classified as Low, as they represent a more detailed analysis of the High and Extreme risks of the High-Level Risk Assessment.

Additionally, the penetration test revealed that the demonstrator discloses a lot of information (see Table 1). The services of the individual ports reveal which (insecure) protocols are used. Not only that the MiR100 is controlled via a HTTP connection, but also that expired certificates and outdated software or protocol standards are used. The Telnet port is not blocked at the access point making a cleartext login possible. Furthermore, the Process Control Server, router and access point are using outdated TLS versions (TLSv1.0 and TLS1.1). Another vulnerability affecting SSL/TLS is the expired certificate on the Process Control Server and the used signature algorithm of the certificate. SHA1 with RSA encryption is now considered weak and should therefore no longer be used. However, these are security breaches in the area of Confidentiality. Using the information gathered, further attacks could be initiated. These resulted in a loss of Integrity, e.g., by sending previously sniffed REST requests to the MiR100. A loss of Availability was triggered either by a Denial of Service (DoS) attack on web interfaces or directly on the Process Control Server. Additionally, a loss of Availability was caused by the execution of different scans (e.g., OpenVAS, nmap (1)) or the manipulation of the MiR100 using the ROSPenTo, a penetration testing tool for ROS [28].

\begin{tabular}{|l|l|c|}
\hline \multicolumn{1}{|c|}{ Vulnerability / Threat } & \multicolumn{1}{|c|}{ Affected Device } & Risk Classification \\
\hline Encryption of the OPC UA communication & $\begin{array}{l}\text { MiRWrapper, Process Control Server } \text { and } \\
\text { HMI }\end{array}$ & Medium \\
\hline Expired SSL/TLS certificate & Process Control Server & Medium \\
\hline Disclosure of the use of insecure services & $\begin{array}{l}\text { Process Control Server, access point and } \\
\text { MiR100 }\end{array}$ & Medium \\
\hline Telnet Unencrypted Login & Access point & High \\
\hline Service failure triggered by an intrusion & MiRWrapper and Process Control Server & High \\
\hline ROSPentTo Stealth Publisher Attack [28] & MiRWrapper and MiR100 & Extreme \\
\hline Choice of insecure passwords for the devices & MiRWrapper and MiR100 \\
\hline
\end{tabular}

Table 2. Identified and most relevant vulnerabilities of the demonstrator, affected devices and respective risk classification

None of the actively conducted attacks resulted in a movement of the MiR100 or a failure of the safety system, primarily because it was not considered to be a potential target. In conclusion, the MiR100 within the demonstrator does not pose a danger related to machine safety per se. Nevertheless, this combination poses a high risk for cyber-attacks.

\section{Conclusion}

By integrating CPS, such as mobile robots, as part of modern production systems, new opportunities for attacks and potential hazards arise. A high level of expertise is required in order to identify and mitigate these with suitable measures. Laws, standards and guidelines, especially in the area of safety, already form a well-developed basis. However, there is also an interaction with IT security. The lack of awareness concerning security in production facilities is still a challenge. Therefore, this study practically analyses the cyber-security threats associated with mobile robotics and the impact of selected attacks on safety. Based on a risk assessment, a penetration test was performed on the demonstrator and the MiR100. It revealed vulnerabilities already identified in the previous step by practical application.

${ }^{9}$ https://www.openvas.org/ 
In particular, the security aspect is endangered by the high vulnerability of Confidentiality. Breaches in the security goals of Integrity and Availability were also revealed which had no impact directly on the safety of the mobile robot, as the critical parts were excluded from the penetration test.

Concluding, it can be said that the MiR100 within the demonstrator does not pose a danger related to machine safety per se. However, this does not apply to other scenarios where hazards for persons in the area around the robot triggered by security breaches may certainly occur. In order to accomplish an integration of mobile robotics in a production environment, a mitigation of the identified risks and a subsequent re-evaluation is necessary. This applies in particular to cyber-security risks, as these are not yet covered by legal regulations. Therefore, it is recommended to apply the IEC 62443 series as a basis for securing industrial communication networks.

Further research can be conducted by the extension with a real production process, consisting of a machine and transported goods. Other and more extensive scenarios can be realised, which may also pose a greater threat to safety. In addition, removing the restrictions for the penetration test (safety PLC, MiR's computer and MiR's internal router) offers three entirely new attack targets, providing a definite greater impact on safety. In addition, the attack surface of Industry 4.0 plants is increasing due to the implementation of additional Cyber-Physical Systems. Therefore, the connection between safety and security cannot be neglected in industry. A general awareness of the connection between safety and security has to be created.

\section{Acknowledgments}

This work was partly supported by the MA23 of the city of Vienna as a part of the project 19-05 "Sicherheit in intelligenten Produktionsumgebungen (SIP4.0)". We would like to express our special thanks to our sponsor MA23 Vienna, Austria.

\section{References}

[1] Martins, G.; Bhatia, S.; Koutsoukos, X.; Stouffer, K.; Tang, C. \& Candell, R. (2015). Towards a systematic threat modeling approach for cyber-physical systems, Proceedings of Resilience Week (RWS), 18-20 Aug. 2015, Philadelphia, PA, USA, ISBN 978-1-4799-8594-4, pp. 1-6, IEEE, DOI: 10.1109/RWEEK.2015.7287428

[2] Papa, M; Kaselautzke, D; Stuja, K \& Wolfel, W (2018). Different Safety Certifiable Concepts for Mobile Robots in Industrial Environments, Proceedings of the 29th DAAAM International Symposium, pp.0791-0800, B. Katalinic (Ed.), Published by DAAAM International, ISBN 978-3-902734-20-4, ISSN 1726-9679, Vienna, Austria DOI: 10.2507/29th.daaam.proceedings. 115

[3] Dieber, B. \& Breitling, B. (2019). Security considerations in modular mobile manipulation, Proceedings of the Third IEEE International Conference on Robotic Computing (IRC), 25-27 Feb. 2019, Naples, Italy, ISBN 978-1-53869245-5, pp. 70-77, IEEE, DOI: 10.1109/IRC.2019.00019

[4] Zimmermann, S.; Reimann, G.; Bock, H.-P.; Ditting, S.; Gernhard, H.-U.; Mehrfeld, J.; Stadler, W.; Straßer, F.; Wittwer, B.-U. \& Wirth, H. (2019). Industrial security in machinery and plant engineering - results of the VDMA study and recommendations for action (Industrial Security im Maschinen- und Anlagenbau Ergebnisse der VDMAStudie und Handlungsempfehlungen), VDMA Competence Center Industrial Security, Frankfurt

[5] International Electrotechnical Commission (2009). IEC/TS 62443-1-1 Ed. 1.0:2009 Industrial communication networks - Network and system security - Part 1-1: Terminology, concepts and models

[6] https://www.tuv.at/next-generation/white-paper/, (2018). White Paper III - safety and security in human-robot collaboration - Influence of IT-security, TÜV AUSTRIA HOLDING AG \& FRAUNHOFER AUSTRIA RESEARCH GMBH, Accessed on: 2021-09-20

[7] https://www.trendmicro.com/vinfo/us/security/news/internet-of-things/rogue-robots-testing-industrial-robotsecurity, (2017). Rogue Robots: Testing the limits of an industrial robot's security, Trend Micro Incorporated., Accessed on: 2021-09-20

[8] Lottenmoser, A.; Berger, C.; Braunreuther, S. \& Reinhart, G. (2017). Method of usability for mobile robotics in a manufacturing environment. Procedia CIRP, Vol. 62 (July 2017) pp. 594-599, ISSN 2212-8271

[9] Siegwart, R. \& Nourbakhsh, N. (2004). Introduction to autonomous mobile robots, MIT Press, ISBN 0-262-19502$\mathrm{X}$, Cambridge, USA

[10] https://www.mobile-industrial-robots.com/en/insights/amr-safety/your-guide-to-a-safe-mobile-robot-installation/, (no date) Your guide to a safe mobile robot installation, Mobile Industrial Robots A/S, Accessed on: 2021-09-20

[11] Clark, G. W.; Doran, M.V. \& Andel, T. R. (2017). Cybersecurity issues in robotics, Proceedings of the IEEE Conference on Cognitive and Computational Aspects of Situation Management (CogSIMA), 27-31 March 2017, Savannah, GA, USA, ISSN 2379-1675, ISBN 978-1-5090-6380-2, pp. 1-5, IEEE, DOI: 10.1109/COGSIMA.2017.7929597

[12] Markis, A.; Papa, M.; Kaselautzke, D.; Rathmair, M.; Sattinger, V. \& Brandstötter, M. (2019). Safety of Mobile Robot Systems in Industrial Applications, Proceedings of the Joint ARW \& OAGM Workshop 2019, 9-10 May 2019, Steyr, Austria, ISBN 978-3-85125-663-5, pp. 26-31, Verlag der Technischen Universität Graz, DOI: $10.3217 / 978-3-85125-663-5-04$ 
[13] https://europa.eu/youreurope/business/product-requirements/standards/standards-in-europe/index_en.htm, (2021). Standards in Europe, European Union, Accessed on: 2021-09-20

[14] Gifei, S. \& Salceanu, A. (2017). Integrated management system for quality, safety and security in developing autonomous vehicles, Proceedings of the 10th International Symposium on Advanced Topics in Electrical Engineering (ATEE), 23-25 March 2017, Bucharest, Romania, ISBN 978-1-5090-5160-1, pp. 673-676, IEEE, DOI: 10.1109/ATEE.2017.7905041

[15] https://www.vdi.de/richtlinien, (2019). VDI guidelines: Setting standards - at the current state of the art (VDIRichtlinien: Standards setzen - auf dem aktuellen Stand der Technik), VDI Verein Deutscher Ingenieure e.V., Accessed on: 2021-09-20

[16] Bostelman, R. V. \& Messina, E. R. (2016). Autonomous industrial vehicles: From the laboratory to the factory floor, National Institute of Standards and Technology (NIST), West Conshohocken, Pennsylvania, USA

[17] Reith, K.; Boden, P.; Däumler, M.; Rank, S.; Schmidt, T. \& Hupfer, R. (2019). Evaluating automated guided vehicle system characteristics in semiconductor fab automated material handling systems, Proceeding of the 30th Annual SEMI Advanced Semiconductor Manufacturing Conference (ASMC), 6-9 May 2019, Saratoga Springs, NY, USA, ISSN 2376-6697, ISBN 978-1-5386-7601-1, pp. 1-6, IEEE, DOI: 10.1109/ASMC.2019.8791758

[18] Capak, T.; Kohar, R.; Škrabala, J. \& Galík. J. (2019). Optimization of traction unit for lowcost automated guided vehicle. IOP Conference Series: Materials Science and Engineering, Vol. 659, No. 1, pp. 012064, ISSN 17578981

[19] Lee, C. K. M.; Keung, K. L.; Ng, K. K. H. \& Lai, D. C. P. (2018). Simulation-based multiple automated guided vehicles considering charging and collision-free requirements in automatic warehouse, Proceedings of the IEEE International Conference on Industrial Engineering and Engineering Management (IEEM), 16-19 Dec. 2018, Bangkok, Thailand, ISSN 2157-362X, ISBN 978-1-5386-6786-6, pp. 1367-1380, IEEE, DOI: 10.1109/IEEM.2018.8607396

[20] Fedorko, G.; Honus, S. \& Salai. R. (2017). Comparison of the traditional and autonomous AGV systems. MATEC Web of Conferences, Vol. 134, pp. 00013, ISSN 2261-236X

[21] Watfa, M. K. \& Karmadi. K. A. (2019). Connected automated guided vehicles in a smart container terminal. OP Conference Series: Materials Science and Engineering, Vol. 659, pp. 012064, ISSN 1757-899X

[22] Breiling, B.; Dieber, B. \& Schartner, P. (2017). Secure communication for the robot operating system, Proceedings of the Annual IEEE International Systems Conference (SysCon), 24-27 April 2017, Montreal, QC, Canada, ISSN 2472-9647, ISBN 978-1-5090-4623-2, pp. 1-6, IEEE, DOI 10.1109/SYSCON.2017.7934755

[23] Taurer, S.; Breiling, B.; Svrta, S. \& Dieber, B. (2019). Case study: Remote attack to disable MiR100 safety, Proceedings of the first Cybersecurity for Robotics 2019 Conference (CSfR2019), Available from: https://www.joanneum.at/robotics/publikationen/detail/case-study-remote-attack-to-disable-mir100-safety Accessed on: 2021-09-20

[24] Reithner, I.; Papa, M.; Lueger, B.; Ćato, M.; Hollerer, S. \& Seemann, R. (2020). Development and implementation of a secure production network, Proceedings of the 31st DAAAM International Symposium, pp. 0736-0745, B. Katalinic (Ed.), Published by DAAAM International, ISBN 978-3-902734-29-7, ISSN 1726-9679, Vienna, Austria DOI: $10.2507 / 31$ st.daaam.proceedings.102

[25] Austrian Association for Electrical Engineering (2018). OVE EN 62443-3-2:2018 Security for industrial automation and control systems - Part 3-2: Security risk assessment and system design

[26] Shebli, H. M. Z. A. \& Beheshti, B. D. (2018). A study on penetration testing process and tools, Proceedings of the IEEE Long Island Systems, Applications and Technology Conference (LISAT), 4 May 2018, Farmingdale, NY, USA, ISBN 978-1-5386-5029-5, pp. 1-7, IEEE, DOI: 10.1109/LISAT.2018.8378035

[27] Barceló, M.; Herzog, P.;Chiesa, R.; Ivaldi, M.; Guasconi, F.; Sensibile, F.; Rudolph, H.; Brown, A.; Mitchell, R.; Feist, R.; Mayencourt, N.; Weber, U.; Gschwend, A.; Bader, T.; Lee, R.-E.; Puente, F.; Truett, K.; Abella, J.; Rosielle, C.; Baumgartner, C. \& Louis, J.-C. (2010) OSSTMM 3 - The Open Source Security Testing Methodology Manual, Institute for Security and Open Methodologies (ISECOM), Cardedeu, Spain

[28] Dieber, B.; White, R.; Taurer, S.; Breiling, B.; Caiazza, G.; Christensen, H. \& Cortesi, A. (2020). Penetration testing ROS, In: Robot Operating System (ROS): The Complete Reference (Volume 4), Springer International Publishing, ISBN 978-3-030-20190-6, Cham, Germany 